\title{
Colourful theorems and indices of homomorphism complexes
}

\author{
Gábor Simonyi* \\ Alfréd Rényi Institute of Mathematics \\ Hungarian Academy of Sciences \\ H-1053 Budapest, Reáltanoda u. 13-15. \\ H-1364 Budapest, P. O. Box: 127 \\ Hungary \\ simonyi.gabor@renyi.mta.hu
}

\author{
Claude Tardif ${ }^{\dagger}$ \\ Royal Military College of Canada \\ PO Box 17000 Station Forces" \\ Kingston, Ontario \\ Canada, K7K 7B4 \\ Claude.Tardif@rmc.ca
}

Submitted: May 1, 2012; Accepted: Jan 7, 2013; Published: Jan 14, 2013

Mathematics Subject Classifications: 05C15, 05C55

\begin{abstract}
We extend the colourful complete bipartite subgraph theorems of [G. Simonyi, G. Tardos, Local chromatic number, Ky Fan's theorem, and circular colorings, Combinatorica 26 (2006), 587-626] and [G. Simonyi, G. Tardos, Colorful subgraphs of Kneser-like graphs, European J. Combin. 28 (2007), 2188-2200] to more general topological settings. We give examples showing that the hypotheses are indeed more general. We use our results to show that the topological bounds on chromatic numbers of digraphs with tree duality are at most one better than the clique number. We investigate combinatorial and complexity-theoretic aspects of relevant order-theoretic maps.
\end{abstract}

Keywords: Topological bounds, Chromatic numbers

*The first author's research is partially supported by the Hungarian Foundation for Scientific Research Grant (OTKA) Nos. K76088 and NK78439

†The second author's research is supported by grants from NSERC and ARP

${ }^{\ddagger}$ The third author’s research is partially supported by the Hungarian Foundation for Scientific Research Grant and by the National Office for Research and Technology (Grant number OTKA 67651) 


\section{Introduction}

For any proper colouring $c$ of a graph $G$ and any linear ordering of the colours, $G$ contains a path with $k=\chi(G)$ vertices all of which have different colours, appearing in increasing order along the path. Indeed this is a consequence of the classical result of Gallai on colourings and orientations: If we orient each edge towards its endpoint with the larger colour, we get an acyclic orientation of a $k$-chromatic graph, which must contain a directed $k$-path, that is, a path with $k$ colours appearing in increasing order. Furthermore if $c$ uses only $k=\chi(G)$ colours, we can say more: According to an exercise in Douglas West's textbook [28], if $T$ is any tree on $k$ vertices labeled by the colours, then $G$ contains a coloured copy of $T$.

These are elementary examples of "colourful subgraph" theorems. The existence of graphs with large girth and large chromatic number shows that trees cannot be replaced by other types of graphs in these results. Also, the existence of graphs with local chromatic number 3 and large chromatic number (see [9]) shows that if $c$ uses more than $k=\chi(G)$ colours, then $G$ is not even guaranteed to contain a claw using four different colours. So these two colourful subgraph results cannot be extended in the general case.

Nonetheless, the context of the local chromatic number prompted the authors of $[24$, $25]$ to investigate analogous results involving colourful complete bipartite subgraphs. They had to restrict their attention to classes of graphs with suitable structural properties. As their results show, effective criteria exist among the topological obstructions to having a small chromatic number, measured by indices and coindices of complexes associated with graphs, in the spirit of $[8,17,18]$.

Theorem 1 (Zig-zag theorem [24]). Let $G$ be a graph such that $\operatorname{coind}\left(B_{0}(G)\right)+1 \geqslant t$. Let $c$ be a proper colouring of $G$ by an arbitrary number of colours. We assume the colours are linearly ordered. Then $G$ contains a complete bipartite subgraph $K_{\left\lceil\frac{t}{2}\right\rceil,\left\lfloor\frac{t}{2}\right\rfloor}$ such that $c$ assigns distinct colours to all $t$ vertices of this subgraph and these colours appear alternating on the two sides of the bipartite subgraph with respect to their order.

The parameter coind $\left(B_{0}(G)\right)$ used in this result is the "coindex of the box complex $B_{0}(G)$ "; for its definition see Section 3. The inequality $\chi(G) \geqslant \operatorname{coind}\left(B_{0}(G)\right)+1$ belongs to a hierarchy of topological lower bounds for $\chi(G)$ that will also be discussed in Section 3. When the bound is tight, more can be said about the structure of colourings:

Theorem 2 (Colourful $K_{l, m}$ theorem [25]). Let $G$ be a graph for which $\chi(G)=$ coind $\left(B_{0}(G)\right)+1=t$. Let $c: V(G) \rightarrow\{1, \ldots, t\}$ be a proper colouring of $G$ and let $A, B \subseteq\{1, \ldots, t\}$ form a bipartition of the colour set, i.e., $A \cup B=\{1, \ldots, t\}$ and $A \cap B=\emptyset$.

Then there exists a complete bipartite subgraph $K_{l, m}$ of $G$ with sides $L, M$ such that $|L|=l=|A|,|M|=m=|B|$, and $\{c(v): v \in L\}=A$, and $\{c(v): v \in M\}=B$. In particular, this $K_{l, m}$ is completely multicoloured by c.

Theorems 1 and 2 were proved using results from algebraic topology, namely Ky Fan's theorem for Theorem 1 and the Tucker-Bacon theorem for Theorem 2 (both of which 
are equivalent versions of the celebrated Borsuk-Ulam theorem, see [2]). Here we present extensions of Theorems 1 and 2 using the parameter $\operatorname{ind}\left(\operatorname{Hom}\left(K_{2}, G\right)\right)+2$ instead of coind $\left(B_{0}(G)\right)+1$. Our extension of Theorem 1 is purely discrete and order-theoretic, while the extension of Theorem 2 (communicated to us by Carsten Schultz) uses the continuum in an essential way.

The paper is structured as follows: In Section 2, we present order-theoretic prerequisites, and an order-theoretic version of Theorem 1. Then we move on to topological spaces in Section 3, where we prove the main results. In Section 4 we apply our results to the class of digraphs with tree duality, to show that for these digraphs all the standard topological lower bounds on the chromatic number give a bound at most one better than the clique number. Finally in Section 5, we explore the complexity aspects of the order-theoretic context.

\section{$2 \quad \mathbb{Z}_{2}$-posets}

Two sets $A, B$ of vertices of a graph $G$ are said to be totally joined if every vertex of $A$ is adjacent to every vertex of $B$. We denote $\operatorname{Hom}\left(K_{2}, G\right)$ the set of ordered couples $(A, B)$ of totally joined nonempty sets of vertices of $G$. There is a natural ordering $\leqslant$ on $\operatorname{Hom}\left(K_{2}, G\right)$, defined by $(A, B) \leqslant\left(A^{\prime}, B^{\prime}\right)$ if $A \subseteq A^{\prime}$ and $B \subseteq B^{\prime}$. We also consider the involution - on $\operatorname{Hom}\left(K_{2}, G\right)$ defined by $-(A, B)=(B, A) \operatorname{Hom}\left(K_{2}, G\right)$ endowed with $\leqslant$ and - is a $\mathbb{Z}_{2}$-poset, that is, an ordered set with a fixed-point free automorphism - of order 2. (Note that we call $\operatorname{Hom}\left(K_{2}, G\right)$ the face poset of the polyhedral complex usually called $\operatorname{Hom}\left(K_{2}, G\right)$ in the literature.) A $\mathbb{Z}_{2}$-map between $\mathbb{Z}_{2}$-posets $P$ and $Q$ is an order-preserving map $\phi: P \rightarrow Q$ such that $\phi(-x)=-\phi(x)$.

The notation $\operatorname{Hom}\left(K_{2}, G\right)$ stands for the homomorphism complex of $K_{2}$ in $G$ : For every $(A, B) \in \operatorname{Hom}\left(K_{2}, G\right)$, we get a homomorphism of the complete graph $K_{2}$ (with vertex-set $\{0,1\})$ to $G$ by selecting any pair of elements $a \in A, b \in B$ as respective images of 0 and 1. For graphs $G$ and $H$, if there exists a homomorphism $\psi: G \rightarrow H$, then we can define a $\mathbb{Z}_{2}$-map $\hat{\psi}: \operatorname{Hom}\left(K_{2}, G\right) \rightarrow \operatorname{Hom}\left(K_{2}, H\right)$ by $\hat{\psi}(A, B)=(\psi(A), \psi(B))$.

For an integer $n \geqslant 0$, let $Q_{n}$ be the $\mathbb{Z}_{2}$-poset on the $2(n+1)$-element set $\pm 0, \pm 1, \ldots, \pm n$, with its natural involution and the order defined by $x<y$ (in $Q_{n}$ ) if $|x|<|y|$ (in $\mathbb{N}$ ). For a $\mathbb{Z}_{2}$-poset $P$, we denote $\operatorname{Xind}(P)$ the smallest $t$ such that $P$ admits a $\mathbb{Z}_{2}$-map to $Q_{t}$. $\operatorname{Xind}(P)$ is called the cross-index of $P$, because of the connection between $Q_{n}$ and the cross-polytope that will be presented in the next section.

Let $K_{n+2}$ be the complete graph with vertex-set $\{0, \ldots, n+1\}$. Then $\operatorname{Hom}\left(K_{2}, K_{n+2}\right)$ is just the set of pairs $(A, B)$ of disjoints nonempty subsets of $V\left(K_{n+2}\right)$. There exists a $\mathbb{Z}_{2}$-map $\phi: \operatorname{Hom}\left(K_{2}, K_{n+2}\right) \rightarrow Q_{n}$ defined by

$$
\phi(A, B)=\left\{\begin{array}{l}
|A \cup B|-2 \text { if } \min (A \cup B) \in A \\
-(|A \cup B|-2) \text { if } \min (A \cup B) \in B .
\end{array}\right.
$$

Therefore, for any graph $G$ with chromatic number $n+2$ and any proper colouring $c$ : $V(G) \rightarrow V\left(K_{n+2}\right), \phi \circ \hat{c}$ is a $\mathbb{Z}_{2}$-map of $\operatorname{Hom}\left(K_{2}, G\right)$ to $Q_{n}$. Thus we have $\chi(G) \geqslant$ $\operatorname{Xind}\left(\operatorname{Hom}\left(K_{2}, G\right)\right)+2$. 
Lemma 3. Let $G$ be a graph such that $\operatorname{Xind}\left(\operatorname{Hom}\left(K_{2}, G\right)\right)+2 \geqslant t$, and let $c$ be a proper colouring of $G$ by an arbitrary number of colours. We assume the colours are linearly ordered. Then $G$ contains a complete bipartite subgraph $K_{\left\lceil\frac{t}{2}\right\rceil,\left\lfloor\frac{t}{2}\right\rfloor}$ such that $c$ assigns distinct colours to all $t$ vertices of this subgraph and these colours appear alternating on the two sides of the bipartite subgraph with respect to their order.

Proof. For $(A, B) \in \operatorname{Hom}\left(K_{2}, G\right)$, there exists a longest sequence $x_{1}, \ldots, x_{\ell}$ alternating between elements of $A$ and elements of $B$, and such that $c\left(x_{1}\right)<\cdots<c\left(x_{\ell}\right)$. We put $\ell(A, B)=\ell, \sigma(A, B)=+$ if $c\left(x_{1}\right)$ is used in $A$, and $\sigma(A, B)=-$ if $c\left(x_{1}\right)$ is used in $B$. (Although the sequence $x_{1}, \ldots, x_{\ell}$ may not be unique, $\sigma(A, B)$ is well defined.) We define $\phi: \operatorname{Hom}\left(K_{2}, G\right) \rightarrow Q_{|V(G)|-2}$ by

$$
\phi(A, B)=\left\{\begin{array}{l}
\ell(A, B)-2 \text { if } \sigma(A, B)=+ \\
-(\ell(A, B)-2) \text { if } \sigma(A, B)=-.
\end{array}\right.
$$

Then $\phi$ is a $\mathbb{Z}_{2}$-map. If $\operatorname{Xind}\left(\operatorname{Hom}\left(K_{2}, G\right)\right)+2 \geqslant t$, then $\phi\left(\operatorname{Hom}\left(K_{2}, G\right)\right) \nsubseteq Q_{t-3}$, hence there exists $(A, B) \in \operatorname{Hom}\left(K_{2}, G\right)$ such that $\phi(A, B) \notin Q_{t-3}$. By definition of $\phi$, there exists a sequence $x_{1}, \ldots, x_{t}$ alternating between elements of $A$ and elements of $B$, and such that $c\left(x_{1}\right)<\cdots<c\left(x_{t}\right)$.

The conclusion of Lemma 3 is the same as that of Theorem 1. In the next section, we will see that $\operatorname{Xind}\left(\operatorname{Hom}\left(K_{2}, G\right)\right)+2 \geqslant \operatorname{coind}\left(B_{0}(G)\right)+1$, hence the hypothesis is more general.

\section{The hierarchy of topological bounds}

To any poset $P$, one can associate a simplicial complex whose simplices are the chains of $P$. We denote $\Delta P$ the geometric realization of this complex, that is, the set of functions $f: P \rightarrow[0,1]$ such that $\{p \in P: f(p)>0\}$ is a chain, and $\sum_{p \in P} f(p)=1$. In particular, $\Delta Q_{n}$ is the $n$-dimensional cross polytope, which is homeomorphic to the $n$-dimensional sphere $S^{n}$ (see [17]). More generally, if $P$ is a $\mathbb{Z}_{2}$-poset, then $\Delta P$ is a $\mathbb{Z}_{2}$-space, that is, a topological space with a continuous fixed-point free involution -. A $\mathbb{Z}_{2}$-map between $\mathbb{Z}_{2}$-spaces $X, Y$ is a continuous map $f: X \rightarrow Y$ such that $f(-x)=-f(x)$. Note that the natural homeomorphism between $\Delta Q_{n}$ and $S^{n}$ is in fact a $\mathbb{Z}_{2}$-homeomorphism.

The index $\operatorname{ind}(X)$ of a $\mathbb{Z}_{2}$-space $X$ is the least $n$ such that there exists a $\mathbb{Z}_{2}$-map from $X$ to $S^{n}$, and its coindex $\operatorname{coind}(X)$ is the largest $n$ such that there exists a $\mathbb{Z}_{2}$-map from $S^{n}$ to $X$. By the Borsuk-Ulam theorem (see [17]), we always have coind $(X) \leqslant \operatorname{ind}(X)$.

Any $\mathbb{Z}_{2}$-map between $\mathbb{Z}_{2}$-posets $P$ and $Q$ lifts naturally to a $\mathbb{Z}_{2}$-map between the $\mathbb{Z}_{2-}$ spaces $\Delta P$ and $\Delta Q$. Therefore since $\Delta Q_{n}$ admits a $\mathbb{Z}_{2}$-homeomorphism to $S^{n}$, we always have $\operatorname{Xind}(P) \geqslant \operatorname{ind}(\Delta P)$. (See Section 5 for examples where this inequality is strict.) We will write ind $(P)$ for ind $(\Delta P)$.

In the case of a graph $G$, Lemma 3 has the following consequence. 
Theorem 4. Let $G$ be a graph such that $\operatorname{ind}\left(\operatorname{Hom}\left(K_{2}, G\right)\right)+2 \geqslant t$, and let $c$ be a proper colouring of $G$ by an arbitrary number of colours. We assume the colours are linearly ordered. Then $G$ contains a complete bipartite subgraph $K_{\left\lceil\frac{t}{2}\right\rceil,\left\lfloor\frac{t}{2}\right\rfloor}$ such that $c$ assigns distinct colours to all $t$ vertices of this subgraph and these colours appear alternating on the two sides of the bipartite subgraph with respect to their order.

To compare this result to Theorem 1, we will need to introduce the parameters corresponding to the box complex $B_{0}(G)$. In a slightly unconventional manner, we will define $B_{0}(G)$ to be $\operatorname{Hom}\left(K_{2}, G^{+}\right)$, where $G^{+}$is the graph obtained from $G$ by adding a universal vertex adjacent to all the vertices of $G$. The reader is referred to $[5,6,7,18]$ for the "conventional" definition of $B_{0}(G)$, and the homotopy equivalence with $B_{0}(G)$ as defined here. The hierarchy of "topological bounds" on the chromatic number of a graph $G$ is the following.

$$
\begin{aligned}
\chi(G) & \geqslant \operatorname{ind}\left(\operatorname{Hom}\left(K_{2}, G\right)\right)+2 \geqslant \operatorname{ind}\left(B_{0}(G)\right)+1 \\
& \geqslant \operatorname{coind}\left(B_{0}(G)\right)+1 \geqslant \operatorname{coind}\left(\operatorname{Hom}\left(K_{2}, G\right)\right)+2 \geqslant \omega(G) .
\end{aligned}
$$

In particular, this implies that ind $\left(\operatorname{Hom}\left(K_{2}, G\right)\right)+2 \geqslant \operatorname{coind}\left(B_{0}(G)\right)+1$, hence Theorem 4 generalises Theorem 1. The same hypothesis can also be used to generalise Theorem 2 . We present below the proof communicated to us by Carsten Schultz [23].

Theorem 5. Let $G$ be a graph for which $\chi(G)=\operatorname{ind}\left(\operatorname{Hom}\left(K_{2}, G\right)\right)+2=t$. Let $c$ : $V(G) \rightarrow\{1, \ldots, t\}$ be a proper colouring of $G$ and let $A, B \subseteq\{1, \ldots, t\}$ form a bipartition of the colour set, i.e., $A \cup B=\{1, \ldots, t\}$ and $A \cap B=\emptyset$.

Then there exists a complete bipartite subgraph $K_{l, m}$ of $G$ with sides $L, M$ such that $|L|=l=|A|,|M|=m=|B|$, and $\{c(v): v \in L\}=A$, and $\{c(v): v \in M\}=B$. In particular, this $K_{l, m}$ is completely multicoloured by c.

Proof. The colouring $c: G \rightarrow K_{t}$ lifts naturally to a $\mathbb{Z}_{2}$-map $\hat{c}: \Delta \operatorname{Hom}\left(K_{2}, G\right) \rightarrow$ $\Delta \operatorname{Hom}\left(K_{2}, K_{t}\right)$. It is known that $\Delta \operatorname{Hom}\left(K_{2}, K_{t}\right)$ is $\mathbb{Z}_{2}$-homeomorphic to the sphere $S_{t-2}$ (see $[1,22])$. Hence, since $\operatorname{ind}\left(\operatorname{Hom}\left(K_{2}, G\right)\right)=t-2, \hat{c}$ must be surjective.

For the partition $A, B$ of $V\left(K_{t}\right)$, the characteristic function $\xi_{(A, B)}$ of the singleton $\{(A, B)\}$ is an element of $\Delta \operatorname{Hom}\left(K_{2}, K_{t}\right)$. Therefore there exists an element $f$ of $\Delta \operatorname{Hom}\left(K_{2}, G\right)$ such that $\hat{c}(f)=\xi_{(A, B)}$. The support of $f$ is a chain $\left(X_{1}, Y_{1}\right), \ldots,\left(X_{\ell}, Y_{\ell}\right)$ in $\operatorname{Hom}\left(K_{2}, G\right)$, and since $\hat{c}(f)=\xi_{(A, B)}$, we have $\left(c\left(X_{i}\right), c\left(Y_{i}\right)\right)=(A, B)$ for $1 \leqslant i \leqslant \ell$. Therefore, there exist $L \subseteq X_{1}, M \subseteq Y_{1}$ such that $|L|=l=|A|,|M|=m=|B|$, and $c(L)=A, c(M)=B$.

According to [8], the condition $\operatorname{ind}\left(\operatorname{Hom}\left(K_{2}, G\right)\right)+2 \geqslant l+m$ alone guarantees the existence of some $K_{l, m}$ in $G$, without reference to colourings. The idea of using the surjectivity of a map lifted from a colouring $c: G \rightarrow K_{t}$ to a $\mathbb{Z}_{2}$-map $\hat{c}$ from the geometric realization of $B_{0}(G)$ to that of $B_{0}\left(K_{t}\right)$ appeared in Xuding Zhu's presentation [29] of some results of $[19,24]$. It can be used to prove the colourful $K_{l, m}$ theorem with the hypothesis $\chi(G)=\operatorname{ind}\left(B_{0}(G)\right)+1=t$. (This was also observed by Carsten Schultz [21].) 
Theorems 4 and 5 both use the hypothesis ind $\left(\operatorname{Hom}\left(K_{2}, G\right)\right)+2 \geqslant t$. It is interesting to note that while the proof of Theorem 5 uses an argument of continuity, the proof of Theorem 4 is essentially discrete, based on the weaker hypothesis $\operatorname{Xind}\left(\operatorname{Hom}\left(K_{2}, G\right)\right)+2 \geqslant$ $t$. In the last section we give examples of posets $P$ such that $\operatorname{Xind}(P)>\operatorname{ind}(P)$, though we do not have an example of a graph $G$ such that $\operatorname{Xind}\left(\operatorname{Hom}\left(K_{2}, G\right)\right)>\operatorname{ind}\left(\operatorname{Hom}\left(K_{2}, G\right)\right)$. In particular, we cannot prove or disprove that the conclusion of Theorem 5 can be derived from the weaker hypothesis $\operatorname{Xind}\left(\operatorname{Hom}\left(K_{2}, G\right)\right)+2 \geqslant t$, or any of its discrete variations (obtained by replacing $Q_{n}$ by other $\mathbb{Z}_{2}$-posets representing spheres). The best we can do in this direction is the following.

Theorem 6. Let $G$ be a graph such that $\chi(G)=\operatorname{Xind}\left(\operatorname{Hom}\left(K_{2}, G\right)\right)+2=2 n \geqslant 4$. Let $c: V(G) \rightarrow\{1, \ldots, 2 n\}$ be a proper colouring of $G$. Then $G$ contains a copy $C$ of $K_{n+1, n-1}$ such that $c$ uses all $2 n$ colours on $C$.

Proof. Suppose that $\chi(G)=2 n$ and $c$ is a $2 n$-colouring of $G$. If there are no copies $C$ of $K_{n+1, n-1}$ such that $c$ uses all $2 n$ colours on $C$, we can define a $\mathbb{Z}_{2}$-map $\phi: \operatorname{Hom}\left(K_{2}, G\right) \rightarrow$ $Q_{2 n-3}$ as follows. We partition $\operatorname{Hom}\left(K_{2}, G\right)$ into three sets

$$
\begin{aligned}
& S_{1}=\{(A, B): \max \{|c(A)|,|c(B)|\} \leqslant n \text { and }|c(A \cup B)| \leqslant 2 n-2\}, \\
& S_{2}=\{(A, B): \max \{|c(A)|,|c(B)|\} \geqslant n+1\}, \\
& S_{3}=\{(A, B):(|c(A)|,|c(B)|) \in\{(n-1, n),(n, n-1),(n, n)\}\} .
\end{aligned}
$$

If $(A, B) \in S_{1}$, we put

$$
\phi(A, B)=\left\{\begin{array}{l}
|c(A \cup B)|-2 \text { if } \min c(A \cup B) \in c(A), \\
-(|c(A \cup B)|-2) \text { if } \min c(A \cup B) \in c(B) .
\end{array}\right.
$$

Note that $\phi$ clearly preserves the order and the involution on $S_{1}$, and $\phi\left(S_{1}\right) \subseteq$ $\{ \pm 0, \ldots, \pm 2 n-4\}$. The remaining elements on $\operatorname{Hom}\left(K_{2}, G\right)$ will be mapped to $\pm(2 n-3)$. If $(A, B) \in S_{2}$, we put

$$
\phi(A, B)=\left\{\begin{array}{l}
2 n-3 \text { if }|c(A)| \geqslant n+1, \\
-(2 n-3) \text { if }|c(B)| \geqslant n+1 .
\end{array}\right.
$$

Clearly, $\phi$ preserves the order and involution on $S_{1} \cup S_{2}$. It remains to define $\phi$ on $S_{3}$. Here we use the hypothesis that there is no colourful copy of $K_{n+1, n-1}$, which implies that no element of $S_{3}$ is below an element of $S_{2}$. For $(A, B) \in S_{3}$, let $\left(X_{A, B}, Y_{A, B}\right)$ be the partition of $\{1, \ldots, 2 n\}$ such that $c(A) \subseteq X_{A, B}, c(B) \subseteq Y_{A, B}$ and $\left|X_{A, B}\right|=\left|Y_{A, B}\right|=n$. Since no copy of $K_{n+1, n-1}$ is multicoloured by $c$, for $\left(A^{\prime}, B^{\prime}\right) \geqslant(A, B) \in S_{3}$ we have $\left(A^{\prime}, B^{\prime}\right) \in S_{3}$, $X_{A^{\prime}, B^{\prime}}=X_{A, B}$ and $Y_{A^{\prime}, B^{\prime}}=Y_{A, B}$. We put

$$
\phi(A, B)=\left\{\begin{array}{l}
2 n-3 \text { if } 1 \in X_{A, B}, \\
-(2 n-3) \text { if } 1 \in Y_{A, B} .
\end{array}\right.
$$

Thus $\phi: \operatorname{Hom}\left(K_{2}, G\right) \rightarrow Q_{2 n-3}$ is a $\mathbb{Z}_{2}$-map, whence $\operatorname{Xind}\left(\operatorname{Hom}\left(K_{2}, G\right)\right) \leqslant 2 n-3$. 
The question whether coind $\left(\operatorname{Hom}\left(K_{2}, G\right)\right)+2 \geqslant 2 k$ implies also the existence of a completely multicoloured $K_{k-1, k+1}$ subgraph of $G$ in every proper colouring is also posed in [27]. Note that although the conclusion in this statement is identical to the conclusion of our Theorem 6, the hypotheses are not comparable: in Theorem 6 the topological condition is weaker but we insist on using the minimum number of colours.

Our last result of this section will show that the hypotheses $\chi(G)=\operatorname{ind}\left(\operatorname{Hom}\left(K_{2}, G\right)\right)+$ 2 and $\chi(G)=\operatorname{ind}\left(B_{0}(G)\right)+1$ are different. Csorba [7] proved that the difference between $\operatorname{ind}\left(\operatorname{Hom}\left(K_{2}, G\right)\right)+2$ and $\operatorname{ind}\left(B_{0}(G)\right)+1$ is at most 1 , and there are graphs $G$ such that $\operatorname{ind}\left(\operatorname{Hom}\left(K_{2}, G\right)\right)+2>\operatorname{ind}\left(B_{0}(G)\right)+1$. (Note that this could still allow the equality $\chi(G)=\operatorname{ind}\left(\operatorname{Hom}\left(K_{2}, G\right)\right)+2$ to never hold for graphs with this property.) No "combinatorial" construction of such graphs is known.

Proposition 7. For every odd integer $t \geqslant 5$ there exists a graph $H_{t}$ such that

$$
t=\chi\left(H_{t}\right)=\operatorname{ind}\left(\operatorname{Hom}\left(K_{2}, H_{t}\right)\right)+2>\operatorname{ind}\left(B_{0}\left(H_{t}\right)\right)+1=t-1 .
$$

Proof. By results of Csorba [4], for every odd $t \geqslant 5$ there exists a graph $G_{t}$ such that $\operatorname{ind}\left(\operatorname{Hom}\left(K_{2}, G_{t}\right)\right)+2=t$ and $\operatorname{ind}\left(B_{0}\left(G_{t}\right)\right)+1=t-1$. However $\chi\left(G_{t}\right)$ could be larger than $t$. (See also [7] for the case $t=5$.)

Let $H_{t}$ be the categorical product $G_{t} \times K_{t}$; that is, the vertex set of $H_{t}$ is $V\left(G_{t}\right) \times V\left(K_{t}\right)$ and the edges of $H_{t}$ join the pairs $\left(u, u^{\prime}\right),\left(v, v^{\prime}\right)$ such that $\{u, v\} \in E\left(G_{t}\right)$ and $\left\{u^{\prime}, v^{\prime}\right\} \in$ $E\left(K_{t}\right)$. Then we have $\chi\left(H_{t}\right) \leqslant t$. In fact, if $\chi\left(G_{t}\right)=t$, then there are homomorphisms both ways between $G_{t}$ and $H_{t}$, hence $\operatorname{ind}\left(\operatorname{Hom}\left(K_{2}, H_{t}\right)\right)=\operatorname{ind}\left(\operatorname{Hom}\left(K_{2}, G_{t}\right)\right)$ and $\operatorname{ind}\left(B_{0}\left(H_{t}\right)\right)=\operatorname{ind}\left(B_{0}\left(G_{t}\right)\right)$.

If $\chi\left(G_{t}\right)>t$, then there is no homomorphism from $G_{t}$ to $K_{t}$, hence no homomorphism from $G_{t}$ to $H_{t}$. However, since $\operatorname{ind}\left(\operatorname{Hom}\left(K_{2}, G_{t}\right)\right)=t-2$, there exists a $\mathbb{Z}_{2}$-map $f$ from the geometric realization of $\operatorname{Hom}\left(K_{2}, G_{t}\right)$ to $S_{t-2}$. Also, there exists a $\mathbb{Z}_{2}$-homeomorphism $g$ from $S_{t-2}$ to the geometric realization of $\operatorname{Hom}\left(K_{2}, K_{t}\right)$. Hence (id, $g \circ f$ ) is a $\mathbb{Z}_{2}$-map from the geometric realization of $\operatorname{Hom}\left(K_{2}, G_{t}\right)$ to that of $\operatorname{Hom}\left(K_{2}, G_{t}\right) \times \operatorname{Hom}\left(K_{2}, K_{t}\right) \subseteq \operatorname{Hom}\left(K_{2}, G_{t} \times K_{t}\right)=\operatorname{Hom}\left(K_{2}, H_{t}\right)$. Since the first projection on $G_{t} \times K_{t}$ is a homomorphism from $H_{t}$ to $G_{t}$, we conclude that there exist $\mathbb{Z}_{2}$-maps both ways between the geometric realizations of $\operatorname{Hom}\left(K_{2}, G_{t}\right)$ and $\operatorname{Hom}\left(K_{2}, H_{t}\right)$, therefore $\operatorname{ind}\left(\operatorname{Hom}\left(K_{2}, H_{t}\right)\right)+2=\operatorname{ind}\left(\operatorname{Hom}\left(K_{2}, G_{t}\right)\right)+2=t$. Since there is a homomorphism from $H_{t}$ to $G_{t}$, we also have $\operatorname{ind}\left(B_{0}\left(H_{t}\right)\right)+1 \leqslant \operatorname{ind}\left(B_{0}\left(G_{t}\right)\right)+1=t-1$.

\section{Tree duality}

For $1 \leqslant k<n$, the shift graph $\delta^{k}\left(\vec{K}_{n}\right)$ is the directed graph whose vertices are the vectors $\left(a_{0}, a_{1}, \ldots, a_{k}\right)$ such that $1 \leqslant a_{0}<a_{1}<\cdots<a_{k} \leqslant n$ and whose arcs join consecutive vectors $\left(a_{0}, a_{1}, \ldots, a_{k}\right),\left(a_{1}, a_{2}, \ldots, a_{k+1}\right)$. Let $S(n, k)$ be the symmetrisation of $\delta^{k}\left(\vec{K}_{n}\right)$, that is, the undirected graph obtained from $\delta^{k}\left(\vec{K}_{n}\right)$ by replacing every arc by an undirected

edge. It is well known (see [14]) that $\chi(S(n, k)) \simeq \log _{2}^{(k)}(n)$; these are "folklore" examples of graphs with arbitrarily large odd girth and chromatic number. In [26] the zig-zag 
theorem is used to show that $\operatorname{coind}\left(B_{0}(S(n, k))\right)+1=3$. Thus our Theorem 4 can be used to show that in fact we have ind $\left(\operatorname{Hom}\left(K_{2}, S(n, k)\right)\right)+2=3$. In this section, we provide a generalisation of this result involving tree duality.

We will consider orientations of graphs. An orientation $\vec{G}$ of a graph $G$ is a directed graph containing one of the $\operatorname{arcs}(u, v),(v, u)$ for each of the edges $[u, v]$ of $G$. A homomorphism of $\vec{G}$ to $\vec{H}$ is a map from $V(G)$ to $V(H)$ that preserves the orientation as well as the adjacency. An oriented graph $\vec{H}$ is said to have tree duality if the following holds.

For any oriented graph $\vec{G}$, either there exists a homomorphism of $\vec{G}$ to $\vec{H}$ or there exists an oriented tree $\vec{T}$ such that $\vec{T}$ admits a homomorphism to $\vec{G}$ but not to $\vec{H}$.

The oriented graphs with tree duality have been characterised in [10]. In particular, the shift graphs have tree duality (see [12]). The result of [26] stating that $\operatorname{coind}\left(B_{0}(S(n, k))\right)+$ $1=3$ can be generalised as follows.

Theorem 8. Let $H$ be a graph which admits an orientation $\vec{H}$ with tree duality. Then

$$
\omega(H) \leqslant \operatorname{ind}\left(\operatorname{Hom}\left(K_{2}, H\right)\right)+2 \leqslant \omega(H)+1 .
$$

Proof. The first inequality is well known (for any $H$ ). The proof of the second inequality uses basic properties of oriented graphs with tree duality. The simplest examples are the transitive tournaments $\left\{\vec{K}_{n}\right\}_{n \geqslant 1}$ : An oriented graph $\vec{G}$ admits a homomorphism to $\vec{K}_{n}$ if and only if there is no homomorphism of $\vec{P}_{n}$ to $\vec{G}$, where $\vec{P}_{n}$ is the directed path with vertices $0,1, \ldots, n$ and $\operatorname{arcs}(0,1), \ldots,(n-1, n)$. Also note that since any oriented tree admits a homomorphism into any directed cycle (i.e., with all edges oriented in the same direction), an oriented graph with tree duality is necessarily acyclic.

Let $H$ be a graph which admits an orientation $\vec{H}$ with tree duality. The oriented trees $\vec{T}$ not admitting a homomorphism to $\vec{H}$ will be called tree obstructions of $\vec{H}$. For $n=\omega(H)$, the copies of $K_{n}$ in $H$ must be oriented transitively in $\vec{H}$. Therefore $\omega(H)$ is the largest $n$ such that $\vec{K}_{n}$ admits a homomorphism to $\vec{H}$. By tree duality of $\vec{H}$, this is the largest $n$ such that no tree obstruction of $\vec{H}$ admits a homomorphism to $\vec{K}_{n}$. By tree duality of $\vec{K}_{n}$ this is the largest $n$ such that every tree obstruction of $\vec{H}$ contains a homomorphic image (that is, a copy) of $\vec{P}_{n}$.

Let $f: V(H) \rightarrow \mathbb{N}$ be a linear extension of $\vec{H}$, that is, a map such that if $(u, v)$ is an arc of $\vec{H}$, then $f(u)<f(v)$. In particular $f$ is a proper vertex-colouring of $H$, hence by Theorem 4, for $t=\operatorname{ind}\left(\operatorname{Hom}\left(K_{2}, H\right)\right)+2, H$ contains a multicoloured copy of $K_{\left\lceil\frac{t}{2}\right\rceil,\left\lfloor\frac{t}{2}\right\rfloor}$, with the colours alternating sides. With the orientation, this is a copy of $\vec{K}_{\left\lceil\frac{t}{2}\right\rceil,\left\lfloor\frac{t}{2}\right\rfloor}$, with vertices $0, \ldots, t-1$ and $\operatorname{arcs}(i, j)$ such that $i<j$ and $i, j$ have different parities. We will show that $\vec{H}$ does not admit a homomorphic image (and in particular a copy) of $\vec{K}_{\left\lceil\frac{n+2}{2}\right\rceil,\left\lfloor\frac{n+2}{2}\right\rfloor}$.

By the above characterization of $n=\omega(H)$, there exists a tree obstruction $\vec{T}$ of $\vec{H}$ which does not contain a homomorphic image of $\vec{P}_{n+1}$. Let $h: \vec{T} \rightarrow \vec{K}_{n+1}$ be a 
homomorphism (with $V\left(K_{n+1}\right)=\{0, \ldots, n\}$ ). Let $c: T \rightarrow\{0,1\}$ be a proper 2-colouring. We can then define a map $g: V(T) \rightarrow V\left(K_{\left\lceil\frac{n+2}{2}\right\rceil,\left\lfloor\frac{n+2}{2}\right\rfloor}\right)$ by

$$
g(u)=\left\{\begin{array}{l}
2 \cdot\left\lceil\frac{h(u)}{2}\right\rceil \text { if } c(u)=0, \\
2 \cdot\left\lfloor\frac{h(u)}{2}\right\rfloor+1 \text { if } c(u)=1 .
\end{array}\right.
$$

It is straightforward to show that $g$ is a homomorphism of $\vec{T}$ to $\vec{K}_{\left\lceil\frac{n+2}{2}\right\rceil,\left\lfloor\frac{n+2}{2}\right\rfloor}$. Therefore $\vec{H}$ does not contain a copy of $\vec{K}_{\left\lceil\frac{n+2}{2}\right\rceil,\left\lfloor\frac{n+2}{2}\right\rfloor}$, hence ind $\left(\operatorname{Hom}\left(K_{2}, H\right)\right)+2 \leqslant n+1$.

In $[20,11]$ it is shown that the gap between the clique number and the chromatic number can be arbitrarily large even for graphs admitting an orientation with tree duality. In [11] good reasons are given to find tight lower bounds on the chromatic numbers of some of these graphs. In this respect, Theorem 8 is bad news, showing that the topological bounds will not be of help. On the other hand, no known efficient procedure answers the problem of determining whether an input graph $H$ satisfies ind $\left(\operatorname{Hom}\left(K_{2}, H\right)\right)+2 \leqslant n$. Perhaps sometimes exhibiting an oriented tree $T$ not admitting a homomorphism to some orientation of $H$ will be the best resource at hand.

\section{Complexity aspects of the cross-index}

Some remarks on complexity aspects of topological lower bounds on the chromatic number can be found in Kozlov's survey paper [15] (at the end of Subsection 1.1.3) and also in his book [16] (on page 295). He mentions that while Lovász's original lower bound expressed in terms of connectivity of a simplicial complex is difficult to compute, another lower bound based on the so-called Stiefel-Whitney characteristic classes is polynomially computable. (The latter bound also depends on a $\mathbb{Z}_{2}$-space and when it is chosen to be $\operatorname{Hom}\left(K_{2}, G\right)$, then it can be expressed as $h\left(\operatorname{Hom}\left(K_{2}, G\right)\right)+2$, where $h\left(\operatorname{Hom}\left(K_{2}, G\right)\right)$ is the so-called Stiefel-Whitney height of $\operatorname{Hom}\left(K_{2}, G\right)$, cf. page 328 in [16]. It is shown on page 123 of [16] that if $X$ is any $\mathbb{Z}_{2}$-space, then coind $(X) \leqslant h(X) \leqslant \operatorname{ind}(X)$ holds.) There is also an ongoing project to compute the $\mathbb{Z}_{2}$-index (see [3]).

For any integer $d$, we can decide whether $\operatorname{Xind}\left(\operatorname{Hom}\left(K_{2}, G\right)\right) \leqslant d$ with a finite computation. Therefore it is natural to ask the computational complexity of this question. It is not clear that the problem is in NP, since the number of elements in the poset $\operatorname{Hom}\left(K_{2}, G\right)$ is exponential in $|V(G)|$. For general posets we prove the following.

Theorem 9. For an integer $d \geqslant 0$, the problem of determining whether an input $\mathbb{Z}_{2}$-poset $P$ satisfies $\operatorname{Xind}(P) \leqslant d$ is polynomial if $d=0$ and NP-complete otherwise.

This does not imply that deciding whether $\operatorname{Xind}\left(\operatorname{Hom}\left(K_{2}, G\right)\right) \leqslant d$ is NP-hard, since the posets used in the reduction are not hom-complexes of graphs. 
Proof of Theorem 9. It is obvious that the problem is in NP, for one can verify that a mapping from the elements of $P$ to the elements of $Q_{d}$ is a $\mathbb{Z}_{2}$-map in time polynomial in the size of $P$.

First, let us examine the case when $d=0$. In this case $Q_{d}$ has only two elements, +0 and -0 , and they are incomparable. Consider the comparability graph of the poset $P$. This is a graph whose vertices are the elements of $P$, and $\{x, y\}$ is an edge if and only if $x<y$ or $y<x$. We claim that the $\mathbb{Z}_{2}$-map to $Q_{0}$ exists if and only if no element $x$ is connected to its mirror image $-x$ by a path in this graph. To prove this, suppose first that there is a $\mathbb{Z}_{2}$-map $\phi$. If now $\{x, y\}$ is an edge then necessarily $\phi(x)=\phi(y)$ (because $Q_{0}$ does not have comparable but unequal elements), so the same must be true for any two path connected vertices $x$ and $y$ as well. However, if there was a path from some element $x$ to $-x$, that would imply that $\phi(x)=\phi(-x)$, but that is a contradiction since we also know $\phi(-x)=-\phi(x)$. Now suppose for the other hand that there is no such path, and we want to construct the $\mathbb{Z}_{2}$-map. For this, notice that $\{x, y\}$ being an edge implies $\{-x,-y\}$ also being an edge, so the connected components of the graph can be grouped into pairs where the pair of a component consists of the mirror image of the vertices of the component. We can then take each such pair of components and let $\phi$ map the vertices of one of them to +0 and the vertices of the other one to -0 . The two required identities are now obvious: $\phi(-x)=-\phi(x)$, and for any $x, y \in P$ if $x<y$ then $\phi(x) \leqslant \phi(y)$ (in fact they are equal). As the graph can be constructed from the poset and the condition of no paths from $x$ to $-x$ can be checked in polynomial time, we have proved that the problem corresponding to $d=0$ is polynomially decidable.

The following consequence of the first part of the proof is worth remembering. The only obstacle that can exclude a $\mathbb{Z}_{2}$-map to $Q_{0}$ is a sequence of elements $x_{0}, y_{0}, x_{1}, y_{1}, \ldots, x_{k-1}, y_{k-1}, x_{k}=-x_{0}$ such that $x_{i}<y_{i}$ and $x_{i+1}<y_{i}$ for each $i$. As a special case, for $k=1$ this obstacle is simply two elements such that $x<y$ and $-x<y$, which is the reason why there is no $Q_{1} \rightarrow Q_{0}$ map.

Now we shall prove that the problem is NP-hard if $d=1$. For this, we give a Karp reduction from the satisfiability problem of boolean expressions in conjunctive normal form (CNF; for the definition and the NP-completeness of this problem, see, e.g., [13]). What this means is that our proof will have three parts: given a boolean formula in CNF, we first construct a $\mathbb{Z}_{2}$-poset $P$ from it in polynomial time, then we show how to construct a $\mathbb{Z}_{2}$-map from $P$ to $Q_{1}$ if we are given an evaluation of the variables that satisfies the formula, and finally we show the reverse construction of such an evaluation from a $\mathbb{Z}_{2}$-map.

To define $P$, we will give the list of its elements and the involution, and we will give some defining relations in the form $x<y$. The partial order $<$ is then understood to be the least defined transitive relation invariant to the involution and satisfying these defining relations (this is analogous to defining a poset with its Hasse diagram). One can compute the full table for this relation from the defining relations by first adding the relation $-x<-y$ for each axiom $x<y$ given, then taking the transitive closure. This computation can indeed be done in polynomial time. It will be obvious from the construction that it generates the list of defining relations from the formula in polynomial 
time, and that the partial order we get is indeed irreflexive. Now if $P$ is given this way, and we have a mapping $\phi$ from $P$ to $Q_{1}$, if we want to verify that this is indeed a $\mathbb{Z}_{2}$-map, it is enough to check two identities: namely that $\phi(-x)=-\phi(x)$ for all $x \in P$, and that $\phi(x) \leqslant \phi(y)$ for each defining relation $x<y$ (i.e., we do not need to check all pairs $x, y$ ).

Let the boolean variables used be $x_{1}, \ldots, x_{N}$, and the formula $C^{1} \wedge \cdots \wedge C^{K}$. Each clause $C^{k}$ has the form $x_{n_{1}} \vee \bar{x}_{n_{2}} \vee \cdots \vee x_{n_{m}}$. Here each variable independently may or may not be negated; the list $n_{i}$ and its length $m$ actually depend on $k$ but we omit that index for readability; and we assume for convenience that no variable occurs twice in any one clause. The poset $P$ we define has four elements for each variable and four more elements for each term in each clause. Namely, for each variable $x_{n}$, we take four elements called $p_{n},-p_{n}, q_{n},-q_{n}$, and for each clause $C^{k}$, we take $4 m$ new elements, namely $r_{1}^{k},-r_{1}^{k}, r_{2}^{k},-r_{2}^{k}, \ldots, r_{m}^{k},-r_{m}^{k}$, and $s_{1}^{k},-s_{1}^{k}, s_{2}^{k},-s_{2}^{k}, \ldots, s_{m}^{k},-s_{m}^{k}$. For defining the partial order, we first need an auxiliary definition. For every variable $x_{n}$, define $T_{n}$ as the set of all elements $r_{i}^{k}$ where the $i$-th term of $C^{k}$ is $x_{n}$, and define $F_{n}$ as the set of all elements $r_{i}^{k}$ where the $i$-th term of $C^{k}$ is $\bar{x}_{n}$. (We depend on the order of terms in the clauses we fixed.) Notice that each of the $r_{i}^{k}$ elements is a member of exactly one of the $2 N$ sets defined here. Now we list all the defining relations of the partial order. Firstly, each variable $x_{n}$ will have two corresponding relations for each occurrence in a term: $t<p_{n}$ and $t<q_{n}$ for each $t \in T_{n}$, and $f<p_{n}$ and $-f<q_{n}$ for each $f \in F_{n}$, respectively. Secondly, each clause $C^{k}$ has two relations corresponding to each of the $m$ terms in it: $s_{i}^{k}<r_{i}^{k}$ for each $1 \leqslant i \leqslant m$; and $s_{i+1}^{k}<r_{i}^{k}$ for each $1 \leqslant i<m$, and additionally $-s_{1}^{k}<r_{m}^{k}$. (One may notice that the two groups contain the same number of relations, in fact each $r_{i}^{k}$ occurs twice in the first group and twice in the second group.)

An example for this construction is shown on the figure, which lists some clauses of the CNF expression we consider, and shows part of the Hasse diagram of the poset, except that we use the convention that each element and its negation is drawn as only one point, and a crossed out edge means $-x<y$ where $x$ is the endpoint of the edge that is lower on the diagram.

Let us examine some properties of this construction. Firstly, (though this does not really help us) notice that there always exists a $\mathbb{Z}_{2}$-map from $P$ to $Q_{2}$ : namely the one that maps $p_{n} \mapsto+2 ; q_{n} \mapsto+2 ; r_{i}^{k} \mapsto+1 ; s_{i}^{k} \mapsto+0$. Now suppose that there also is a $\mathbb{Z}_{2}$-map $\phi: P \rightarrow Q_{1}$. Observe that we may assume that this takes any $p_{n}$ or $q_{n}$ to \pm 1 without loss of generality: indeed it is easy to amend $\phi$ to have this property by changing the image of such an element from \pm 0 to +1 , and changing the image of its mirror image to -1 accordingly. Similarly, we may assume that any $s_{i}^{k}$ is always brought to \pm 0 . Now observe that for any fixed $k$, at least one of the elements $r_{1}^{k}, r_{2}^{k}, \ldots, r_{m}^{k}$ must be mapped to \pm 1 : indeed in the sequence $s_{1}^{k}, r_{1}^{k}, s_{2}^{k}, r_{2}^{k}, \ldots, s_{m}^{k}, r_{m}^{k}$ each element is comparable to the next one, and together with $-s_{1}^{k}<r_{m}^{k}$ they form the exact kind of obstacle we mentioned that makes it impossible to map all these points to \pm 0 . Finally fix any $n$, and observe that if $\phi\left(p_{n}\right)=\phi\left(q_{n}\right)$ then all elements $f$ of $F_{n}$ must be mapped to \pm 0 , for we must keep both $\phi(f) \leqslant \phi\left(p_{n}\right)$ and $\phi(-f) \leqslant \phi\left(q_{n}\right)$. Similarly if $\phi\left(p_{n}\right) \neq \phi\left(q_{n}\right)$ then $\phi$ maps all elements of $T_{n}$ to \pm 0 (the signs may vary). 


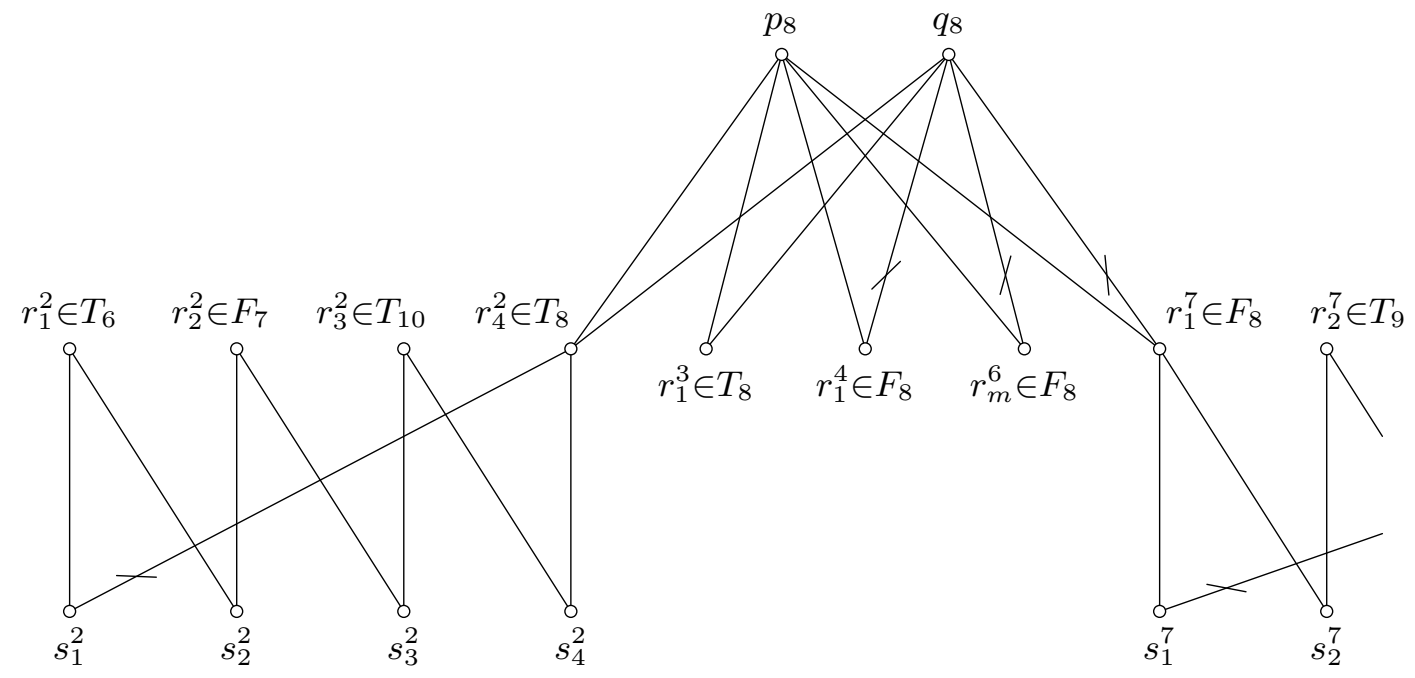

Figure 1: Part of the $\mathbb{Z}_{2}$-poset constructed from a formula some of whose clauses are: $C^{2}=\left(x_{6} \vee \bar{x}_{7} \vee x_{10} \vee x_{8}\right) ; C^{3}=\left(x_{8} \vee \ldots\right) ; C^{4}=\left(\bar{x}_{8} \vee \ldots\right) ; C^{6}=\left(\cdots \vee \bar{x}_{8}\right) ; C^{7}=$ $\left(\bar{x}_{8} \vee x_{9} \vee \ldots\right)$.

This suggests how to derive an evaluation $\sigma$ of the variables from the $\mathbb{Z}_{2}$-map $\phi: P \rightarrow$ $Q_{1}$ : for any $n$, if $\phi\left(p_{n}\right)=\phi\left(q_{n}\right)$ then let $x_{n}^{\sigma}$ be true, otherwise $\phi\left(p_{n}\right)=-\phi\left(q_{n}\right)$ and let $x_{n}^{\sigma}$ be false. Then $\phi$ takes all members of $F_{n}$ to \pm 0 in the former case and all members of $T_{n}$ to \pm 0 in the latter. Consider any clause $C^{k}$ and the elements corresponding to it: we have observed that for at least one $j$, the element $r_{j}^{k}$ is not mapped to \pm 0 . If the term corresponding to this index $j$ in $C^{k}$ is $x_{n}$ then this element $r_{j}^{k} \in T_{n}$, so together with the above this means $x_{n}$ is true; whereas if that term is $\bar{x}_{n}$ then similarly $r_{j}^{k} \in F_{n}$ which implies $x_{n}$ is false. In either case, we have found a term in the clause $C^{k}$ that is true in $\sigma$, and this can be repeated for each clause, thus $\sigma$ indeed satisfies the boolean expression.

Now we assume an evaluation $\sigma$ of the variables $x_{1}, \ldots, x_{N}$ is given and satisfies the formula. We construct a $\mathbb{Z}_{2}$-map $\phi: P \rightarrow Q_{1}$ the following way. Let

$$
\begin{aligned}
& p_{n} \mapsto+1, \quad q_{n} \mapsto+1, \quad \text { if } x_{n}^{\sigma} \text { is true; but } \\
& p_{n} \mapsto+1, \quad q_{n} \mapsto-1, \quad \text { if } x_{n}^{\sigma} \text { is false. }
\end{aligned}
$$

Consider a clause $C^{k}$. The evaluation $\sigma$ satisfies this clause, so at least one of its terms $x_{n_{1}}, \bar{x}_{n_{2}}, \ldots, x_{n_{m}}$ must be evaluated to true: so choose $j$ to be an index of one such term $x_{n_{j}}$ or $\bar{x}_{n_{j}}$. Let $\phi$ act on the elements corresponding to this clause the following way.

$$
\begin{aligned}
& r_{1}^{k} \mapsto+0, \ldots, \quad r_{j-1}^{k} \mapsto+0, \quad r_{j}^{k} \mapsto+1, \quad r_{j+1}^{k} \mapsto-0, \quad \ldots, \quad r_{m}^{k} \mapsto-0 ; \\
& s_{1}^{k} \mapsto+0, \quad \ldots, \quad s_{j-1}^{k} \mapsto+0, \quad s_{j}^{k} \mapsto+0, \quad s_{j+1}^{k} \mapsto-0, \quad \ldots, \quad s_{m}^{k} \mapsto-0 .
\end{aligned}
$$

It is easy to see that these latter assignments satisfy the requirements that $\phi\left(s_{i}^{k}\right) \leqslant$ $\phi\left(r_{i}^{k}\right)$ and $\phi\left(s_{i+1}^{k}\right) \leqslant \phi\left(r_{i}^{k}\right)$ and $-\phi\left(s_{1}^{k}\right) \leqslant \phi\left(r_{m}^{k}\right)$. We must now check the restrictions given by the first group of defining relations of $P$. These, for elements of $T_{n}$, are that 
$\phi\left(r_{i}^{k}\right) \leqslant \phi\left(p_{n_{i}}\right)$ and $\phi\left(r_{i}^{k}\right) \leqslant \phi\left(q_{n_{i}}\right)$ if the $i$-th term of the clause $C^{k}$ is $x_{n_{i}}$. If $i \neq j$ then these are satisfied automatically, because then $\phi\left(r_{i}^{k}\right)= \pm 0$. If, however, $i=j$, then use the fact that we chose $j$ such that $x_{n_{j}}$ is true, thus $\phi\left(p_{n_{i}}\right)=\phi\left(q_{n_{i}}\right)=\phi\left(r_{i}^{k}\right)=+1$. The defining relations involving the elements of $F_{n}$ can be verified in a very similar way: if the $i$-th term of $C^{k}$ is $\bar{x}_{n_{i}}$ then we need $\phi\left(r_{i}^{k}\right) \leqslant \phi\left(p_{n_{i}}\right)$ and $-\phi\left(r_{i}^{k}\right) \leqslant \phi\left(q_{n_{i}}\right)$, but $\phi\left(r_{i}^{k}\right)= \pm 0$ unless $i=j$, in which case $x_{n_{i}}$ is false because of the choice of $j$, so $\phi\left(p_{n_{i}}\right)=\phi\left(r_{i}^{k}\right)=+1$ and $\phi\left(q_{n_{i}}\right)=-1$ satisfy the restrictions. This proves that $\phi$ is indeed a $\mathbb{Z}_{2}$-map.

All that remains now is to prove that the cases of $1<d$ are also NP-complete. This we do by modifying the above Karp reduction. The simple observation we need for this is the following: if we modify any $\mathbb{Z}_{2}$-poset $P$ by adding two extra elements $y$ and $-y$ that are greater than all other elements of the poset, then the cross-index of the resulting $\mathbb{Z}_{2}$-poset $P^{*}$ is exactly one greater than the cross-index of the original. Indeed, we can extend a $P \rightarrow Q_{d}$ map to a $P^{*} \rightarrow Q_{d+1}$ map by setting the image of $y$ to be $+(d+1)$; and conversely, by any $P^{*} \rightarrow Q_{d+1}$ map, no point other than $y$ or $-y$ can be mapped to $\pm(d+1)$, thus restricting it to $P$ gives a $P \rightarrow Q_{d}$ map. Thus, applying the reduction given in the $d=1$ case then iterating this transformation $d-1$ times gives a $\mathbb{Z}_{2}$-poset that can be mapped to $Q_{d}$ if and only if the original expression is satisfiable, and this construction still can be realized by a polynomial time computation.

Remark. Let $P$ be a poset constructed from a boolean formula as in the above proof. Then $\operatorname{Xind}(P)$ depends on the satisfiability of the corresponding boolean formula. However one can prove that ind $(\Delta P)$ is always at most 1 , because any two dimensional face appearing in $\Delta P$ is a triangle which has at least one side that does not belong to any

other two dimensional face. This makes it possible to retract $\Delta P$ into a 1-dimensional complex.

\section{Acknowledgements}

The results discussed above are supported by the grant TÁMOP - 4.2.2.B-10/1-2010-0009.

\section{References}

[1] E. Babson, D. N. Kozlov, Complexes of graph homomorphisms, Israel J. Math., 152 (2006), 285-312.

[2] P. Bacon, Equivalent formulations of the Borsuk-Ulam theorem, Canad. J. Math., 18 (1966), 492-502.

[3] M. Cadek, M. Krcal, J. Matoušek, F. Sergeraert, L. Vokrinek, U. Wagner, Computing all maps into a sphere, preprint (2011) arXiv:1105.6257v3.

[4] P. Csorba, Non-tidy spaces and graph colorings, Ph. D. thesis, ETH Zürich, 2005.

[5] P. Csorba, On the simple $\mathbb{Z}_{2}$-homotopy types of graph complexes and their simple $\mathbb{Z}_{2}$-universality, Canad. Math. Bull., 51 (2008), 535-544. 
[6] P. Csorba, Fold and Mycielskian on homomorphism complexes, Contrib. Discrete Math., 3 (2008), 1-8.

[7] P. Csorba, Homotopy types of box complexes, Combinatorica, 27 (2007), 669-682.

[8] P. Csorba, C. Lange, I. Schurr, A. Wassmer, Box complexes, neighborhood complexes, and the chromatic number, J. Combin. Theory Ser. A, 108 (2004), 159-168.

[9] P. Erdős, Z. Füredi, A. Hajnal, P. Komjáth, V. Rödl, Á. Seress, Coloring graphs with locally few colors, Discrete Math., 59 (1986), 21-34.

[10] T. Feder, M. Vardi, The computational structure of monotone monadic SNP and constraint satisfaction: A study through datalog and group theory. SIAM J. of Computing 28 (1998), 57-104.

[11] J. Foniok, J. Nešetřil, C. Tardif, Interleaved adjoints of directed graphs, European J. Combin. 32 (2011), 1018-1024.

[12] J. Foniok, C. Tardif, Adjoint functors and tree duality, Discrete Math. Theor. Comput. Sci., 11 (2009), 97-110.

[13] M. R. Garey, D. S. Johnson, Computers and intractability: a guide to the theory of NP-completeness, Freeman, New York, 1979.

[14] P. Hell, J. Nešetřil, Graphs and homomorphisms, Oxford Lecture Series in Mathematics and its Applications, 28. Oxford University Press, Oxford, 2004.

[15] D. N. Kozlov, Chromatic numbers, morphism complexes, and Stiefel-Whitney characteristic classes, in: Geometric combinatorics, IAS/Park City Math. Ser., 13, Amer. Math. Soc., Providence, RI, 2007, 249-315, arXiv:math.AT/0505563

[16] D. N. Kozlov, Combinatorial Algebraic Topology, Algorithms and Computation in Mathematics, 21. Springer, Berlin, 2008.

[17] J. Matoušek, Using the Borsuk-Ulam theorem, Lectures on topological methods in combinatorics and geometry, Written in cooperation with Anders Björner and Günter M. Ziegler, Universitext, Springer-Verlag, Berlin, 2003.

[18] J. Matoušek, G. Ziegler, Topological lower bounds for the chromatic number: a hierarchy, Jahresber. Deutsch. Math.-Verein., 106 (2004), 71-90.

[19] F. Meunier, A topological lower bound for the circular chromatic number of Schrijver graphs, J. Graph Theory, 49 (2005), 257-261.

[20] J. Nešetřil, C. Tardif, Path homomorphisms, graph colourings and boolean matrices, J. Graph Theory, 63 (2010), 198-209.

[21] C. Schultz, private communication (Oberwolfach, 2007).

[22] C. Schultz, A short proof of $w_{1}^{n}\left(\operatorname{Hom}\left(C_{2 r+1}, K_{n+2}\right)\right)=0$ for all $n$ and a graph colouring theorem by Babson and Kozlov, Israel J. Math., 170 (2009), 125-134.

[23] C. Schultz, private communication (2011).

[24] G. Simonyi, G. Tardos, Local chromatic number, Ky Fan's theorem, and circular colorings, Combinatorica, 26 (2006), 587-626. 
[25] G. Simonyi, G. Tardos, Colorful subgraphs of Kneser-like graphs, European J. Combin., 28 (2007), 2188-2200.

[26] G. Simonyi, G. Tardos, On directed local chromatic number, shift graphs, and Borsuk-like graphs, J. Graph Theory, 66 (2011), 65-82.

[27] G. Simonyi, G. Tardos, S. T. Vrećica, Local chromatic number and distinguishing the strength of topological obstructions, Trans. Amer. Math. Soc., 361 (2009), 889-908.

[28] D. B. West, Introduction to graph theory, Prentice Hall, Upper Saddle River, NJ, 1996.

[29] X. Zhu, Recent developments in circular colouring of graphs, in: Topics in Discrete Mathematics, Algorithms Combin., 26, Springer, Berlin, 2006, 497-550. 